\title{
1. Arts in/as Therapeutic Practice
}

1.1. The Poem by Beth McGuinness

1.2. Yarn Wrapping Sticks by Jacinta McAvoy

1.3. Double Guru by Amanda Levey and Rachel Grimwood

1.4. A Living Quilt by MAAT Year 3 Whitecliffe Students

1.5. The Imperative for a Daily Reflective Practice by Nona Cameron

1.6. Making Sense of Poietic Presense by Deborah Green

1.7. If I Could Write Poetry by Daniela Gonzalez-Bea

Deb, the thread which feels most alive in the first group is the use of arts in/as therapeutic practice. There are here poetic reflections, journaling practice, yarn wrapping and companioned engagement with/in the natural world; students creatively reflecting upon their experiences of learning therapeutic arts' practice and making sense of that in a creative response. Finally, there are two articles that focus on the value of therapeutic reflective practice in support of maintaining and holding multiple roles of therapist, researcher and educator.

Lacing these pieces together, Stacey, I spy a theme relating to how we centre ourselves in our arts making and arts-based work. I notice, as I read, that my eye is snagged by ideas such as using the arts to land in a space - to playfully layer, to widen the field of play, to multi-voice, to be with hauntings through poiesis, to decentre and incubate and to find words to awaken the magic of the everyday. 